\title{
A case report and review of clinical and laboratory pointers of leukemia in children with bone pain
}

\author{
Elpis Mantadakis, Anastassios Valsamidis \\ Athanassios Chatzimichael
}

\begin{abstract}
Introduction: About 20-30\% of children with acute lymphoblastic leukemia (ALL) present with bone pain, mainly in the lower extremities that can lead to limping or refusal to walk. These children suffer almost exclusively from B-precursor ALL and often present with a nearly normal complete blood count with few or no circulating lymphoblasts. Moreover, they are frequently misdiagnosed as suffering from juvenile arthritis. Case Report: We present case of a 5-year-old boy with mild skeletal complaints for one month who was diagnosed with ALL despite a normal physical examination and minimally affected complete blood count (mild anemia and lymphocytosis alone, with a normal platelet count). Conclusion: Children with ALL and musculoskeletal complaints may have a deceptively normal physical examination at presentation, absence of circulating blasts, and few if any abnormalities on the initial hemogram. Clinical and laboratory pointers to leukemia include bone tenderness or pain,
\end{abstract}

\begin{tabular}{|c|}
\hline $\begin{array}{l}\text { Elpis Mantadakis }{ }^{1} \text { Anastassios } \\
\text { Athanassios Chatzimichael }{ }^{1} \\
\text { Affiliations: }{ }^{1} \text { Department of Pediatrics, Democritus } \\
\text { University of Thrace and University General District } \\
\text { Hospital of Alexandroupolis, Thrace, Greece } \\
\text { Corresponding Author: Elpis Mantadakis, Assistant }\end{array}$ \\
\hline $\begin{array}{l}\text { Professor of Pediatrics, Democritus University of Thrace } \\
\text { and University General District Hospital of } \\
\text { Alexandroupolis, } 6^{\text {th }} \text { Kilometre Alexandroupolis-Makris, } \\
68100 \text { Alexandroupolis, Thrace, Greece; Phone: +30- } \\
\text { 25510-74411; Fax: }+30-25510-30340 ; \quad \text { E-mail: } \\
\text { emantada@med.duth.gr }\end{array}$ \\
\hline $\begin{array}{l}\text { Received: } 2 \text { September } 2010 \\
\text { Accepted: } 19 \text { October } 2010 \\
\text { Published: } 19 \text { December } 2010\end{array}$ \\
\hline
\end{tabular}

especially if nocturnal and non-articular, anemia of any degree, low-normal leukocytes with relative lymphocytosis, red cell macrocytosis, low-normal platelet counts, especially when associated with elevated erythrocyte sedimentation rate and increased serum LDH. Extreme care is needed in the clinical and laboratory evaluation of children with leukemia and skeletal complaints in order not to miss the underlying diagnosis, misdiagnose juvenile arthritis and/or administer corticosteroids.

Keywords: Leukemia, Juvenile arthritis, Skeletal complaints, Differential diagnosis

$$
* * * * * * * * *
$$

Mantadakis E, Valsamidis A, Chatzimichael A. A case report and review of clinical and laboratory pointers of leukemia in children with bone pain. International Journal of Case Reports and Images 2010;1(4):1-6.

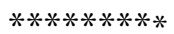

doi:10.5348/ijcri-2010-12-9-CR-1

\section{INTRODUCTION}

Acute lymphoblastic leukemia (ALL) is the most common paediatric malignancy comprising approximately $35 \%$ of all childhood cancers [1]. About 20-30\% of children with ALL present with severe bone pain, mainly in the lower extremities, that can lead to limping or refusal to walk. Sometimes, the skeletal complaints are very subtle but persistent. These children suffer almost exclusively from Bprecursor ALL and often present with a nearly normal complete blood count with few or no circulating lymphoblasts [2]. 
We describe a 5-year-old boy with ALL who presented with mild lower extremities bone pain and night-time fever and was initially thought to be suffering from juvenile arthritis. We review the relevant medical literature with emphasis in clinical and laboratory characteristics that help physicians differentiate ALL from juvenile arthritis.

\section{CASE REPORT}

A 5-year-old boy was referred to us from his pediatrician because of left lower extremity pain that started four weeks ago. Approximately at the same time, he developed night-time low grade fever up to $38.4^{\circ} \mathrm{C}$. Because of the pain, he was initially seen by an orthopaedic surgeon who prescribed tolfenamic acid and who also requested measurement of serum antinuclear antibodies (ANA) that were undetectable. Due to persistence of the complaints, he visited his pediatrician who performed erythrocyte sedimentation rate (ESR) determination that was $120 \mathrm{~mm} / \mathrm{hr}$. Because of the three-digit ESR, he was referred to us with a presumptive diagnosis of a rheumatic disease.

On initial physical examination, the child looked healthy, had normal vital signs, and was in no apparent distress but was complaining of mild deepseated pain in the middle of the left femur. This was the location of the pain from the beginning. Both hip and knee joints were pain-free and meticulous physical examination did not show any point skeletal

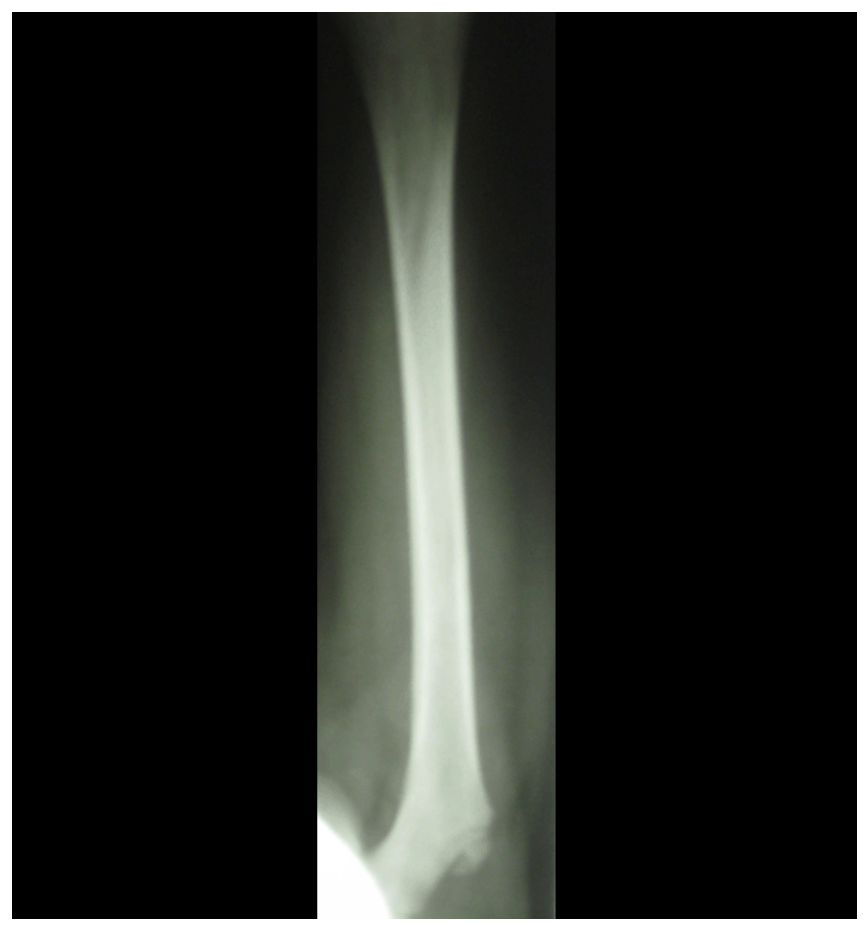

Figure 1. Plain radiograph of the left femur showing no radiographic abnormalities. tenderness. Moreover, he was able to walk and run with minimal discomfort. He had no petechiae, purpura, hepatosplenomegaly or noteworthy lymphadenopathy. Personal history was negative for recent travel, pet or animal contact. Family history was non-contributory. The admission hemogram

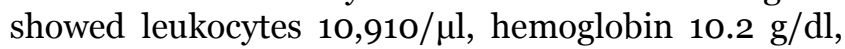
erythrocytes $4.38 \times 10^{6} / \mu \mathrm{l}$, MCV $71 \mathrm{fl}, \mathrm{MCH} 23.3 \mathrm{pg}$, RDW $14.2 \%$, platelets $344,000 / \mu \mathrm{l}$ and reticulocytes $0.51 \%$. Examination of the peripheral blood smear showed $17 \%$ neutrophils, $2 \%$ bands, $1 \%$ myelocytes, $80 \%$ lymphocytes, mild hypochromia and microcytosis with absence of polychromasia, while no blasts were seen. The C-reactive protein was $2.05 \mathrm{mg} / \mathrm{dl}$ (normal <0.5). Serum chemistries were normal except for lactate dehydrogenase (LDH) $389 \mathrm{U} / \mathrm{l}$ (normal <248). A plain radiograph of the left femur (Figure 1) and a chest radiograph (Figure 2) were normal. The patient underwent a bone marrow aspiration that showed $>90 \%$ infiltration by $\mathrm{CD} 10+$, $\mathrm{CD} 38+, \mathrm{DR}+$ lymphoblasts (Figures $3 \mathrm{a}$ and $3 \mathrm{~b}$ ). He is currently undergoing chemotherapy for B-precursor acute lymphoblastic leukemia (ALL).

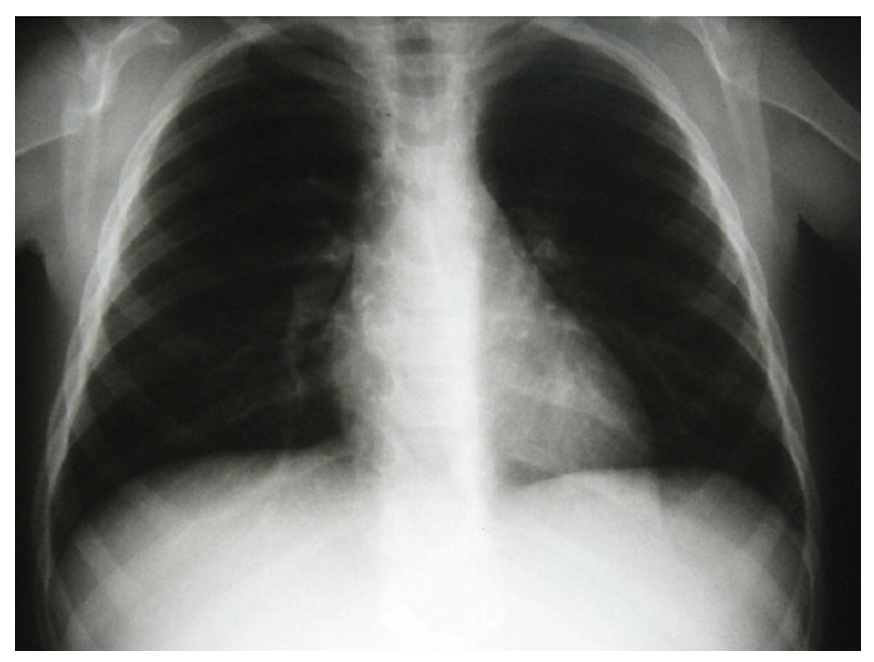

Figure 2. Chest radiograph showing no radiographic abnormalities.

\section{DISCUSSION}

Skeletal complaints are common in growing children. When fever occurs along with bone pain, the differential diagnosis is broad and includes infections, rheumatic conditions, and malignancies.

Children with skeletal complaints and young age ( $<6$ years), self-imposed activity limitations, abnormal neurological examination at any age, night-time exacerbation, and persistent symptoms, especially when associated with fever and/or weight loss should be evaluated for serious underlying pathology [3]. 


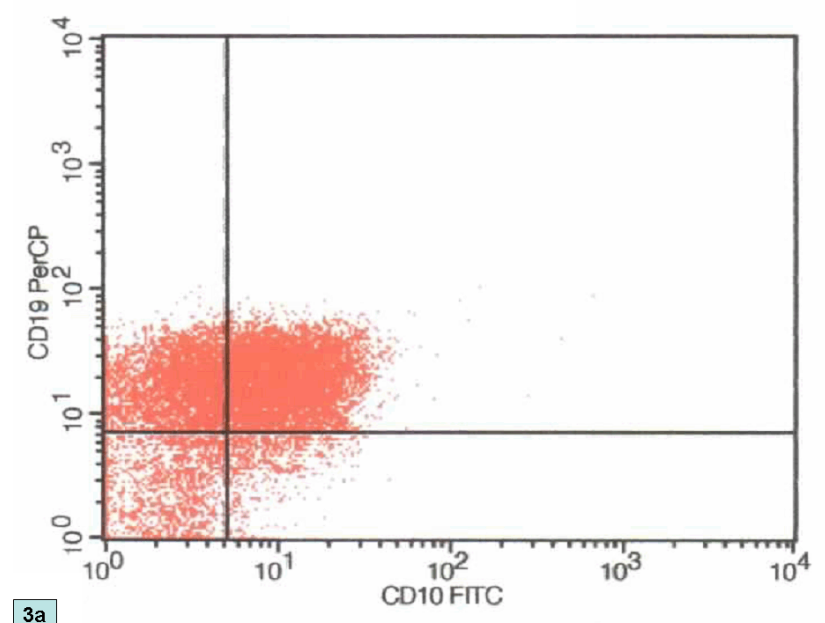

$3 a$

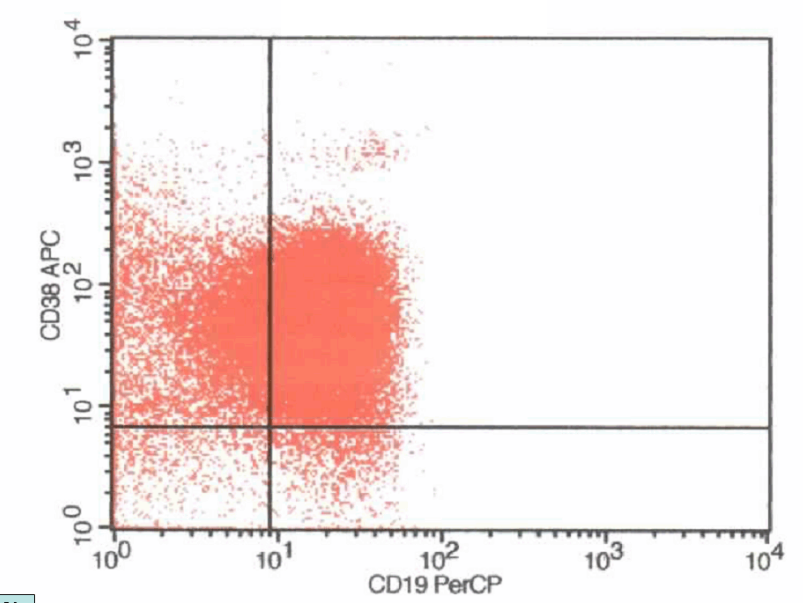

3b

Figure 3. a) Bone marrow flow cytometry showing coexpression of CD10 and CD19 and, b) CD38 and CD19 in the malignant lymphoblast population (B-precursor acute lymphoblastic leukemia).

In any child with skeletal complaints it is important to distinguish true arthritis, i.e., joint inflammation from other muscle and bone problems that do not affect joints. A warm, red and tender joint in a febrile child suggests septic arthritis or rheumatic fever. The septic joint is usually single (monoarticular involvement); the affected child guards it, since even passive movement intensifies pain. On the other hand, diffuse bone pain, especially at night-time points towards malignancy such as leukemialymphoma, or metastatic neuroblastoma. Bone neoplasms, such as Ewing sarcoma and osteosarcoma also cause pain, which however, usually remains localized and is associated with abnormal imaging findings.

Limping along with morning "stiffness" more likely presents in juvenile rheumatoid arthritis (JRA), a condition in which the symptoms persist for at least six weeks, whereas evening symptoms are more indicative of an orthopedic or neurologic problem, the underlying origin of which can be a malignancy.
Sudden appearance of limping suggests traumatic or less commonly infectious etiology. In the latter case, septic fever is usually present. On the other hand, insidious skeletal pains that gradually lead to limping are of greater concern, and are often indicative of a serious underlying neuromuscular disease. This is more likely if there is associated regression in a toddler's developmental milestones.

Although our patient was not febrile at presentation to us, he had history of low grade fever at night-time for the last month, i.e., approximately at the same time that the skeletal complaints began. Although children are less likely to have malignancies or autoimmune disorders and more likely to have infections than adults as the cause of their prolonged fevers, when leukemia or lymphoma is the culprit, it is usually evident by appropriate imaging studies and/or bone marrow examination, like in our case [4].

Regarding ALL, which was the final diagnosis of our patient, bone and joint involvement may be prominent at presentation, and when this occurs misdiagnosis with arthritic conditions is not uncommon [5-7]. About 20-30\% of children with ALL present with severe bone pain, mainly in the lower extremities, and this can lead to limping or refusal to walk. Sometimes the skeletal complaints are subtle but persistent. These children suffer almost exclusively from B-precursor ALL and often present with a nearly normal complete blood count with few or no circulating lymphoblasts, like our patient. Hence, a high index of suspicion is required in order to reach a correct and timely diagnosis.

Age and sex can not help as pointers of juvenile arthritis versus acute leukemia with prominent osteoarticular symptoms. Although ALL in children $<15$ years old is slightly more common in boys (male to female ratio of 1.2) [8], while juvenile arthritis is more common in girls [9], both sexes and all pediatric age groups can be affected by both conditions.

Wallendal et. al. [10] compared a group of 12 children initially thought to have rheumatoid arthritis, but who were eventually diagnosed with a malignant neoplasm, mainly ALL (8 of 12), with a group of children with JRA. At the time of initial presentation, no significant difference was noted in hemoglobin, white blood cell or platelet counts between the two groups. In both groups ESR was elevated, but this elevation was not significantly different. However, serum LDH in the group of children with malignancy was 2.2 times higher than normal versus 0.8 times normal for the group of children with JRA, a significant difference. Our case substantiates the value of serum LDH as a pointer of leukemia.

Our patient had a negative test for ANA. Chudwin et. al. [11] found that $14 \%$ of their referral population with a positive ANA test had other than autoimmune or rheumatic causes for the positive test. Only one among their patients had leukemia. Hence, although children with leukemia do not usually develop autoantibodies, the presence of a positive ANA test 
can not rule out leukemia.

Jones et. al. [12] performed a case-control retrospective chart review to compare the clinical and laboratory findings of 277 children at presentation to a pediatric rheumatology clinic who were ultimately diagnosed with either JRA $(n=206)$ or ALL $(n=71)$. The majority (75\%) of children with ALL did not have circulating lymphoblasts at the time of initial evaluation. In children presenting with unexplained musculoskeletal complaints, the three most important factors that predicted a diagnosis of ALL were low white blood cell count $(<4,000 / \mu l)$, low-normal platelets $(150-250,000 / \mu \mathrm{l})$, and history of night-time pain. In the presence of these three factors, the sensitivity and specificity for a diagnosis of ALL were $100 \%$ and $85 \%$, respectively. ANA and clinical signs of arthritis were not helpful in differentiating diagnoses of JRA from ALL. In the same study, apart from these three factors, low hemoglobin and high serum LDH were also significantly different among patients with JRA and ALL.

Our child had a normal total leukocyte count and platelets were towards the upper limit of normal $(344,000 / \mu l)$. Blatt et. al. [13] described 7 children, all boys, out of 217 (3.2\%) with ALL who had platelet counts $>400,000 / \mu \mathrm{l}$ at diagnosis. Other than male sex, no clinical or laboratory characteristics were clearly associated with thrombocytosis. Thus, although the platelet count is usually decreased at diagnosis of pediatric ALL, it can be normal or even elevated.

Jonsson et. al. [14] reviewed the medical records of children with ALL, diagnosed and treated in a single centre over a 13-year period to determine the relationship between bone pain and hematologic findings at diagnosis. Of 296 children with ALL, 179 children (60\%) had no bone pain, 65 children (22\%) had some bone pain, and 52 children (18\%) had prominent bone pain that overshadowed other leukemia manifestations. No differences were detected in the age at diagnosis or gender. Children in the group with intense bone pain had values closer to normal for hemoglobin, leukocyte count, absolute neutrophil count, percentage of circulating lymphoblasts, and platelets than those in the other ALL subgroups. Moreover, children with ALL and prominent bone pain had symptoms for an average of $>2$ weeks longer prior to diagnosis. Ostrov et. al. [15] studied 10 children with acute leukemia and an equal number of children with systemic JRA (sJRA). Highspiking fever, morning stiffness, and rash were reported by 80 to $90 \%$ of children with sJRA and in only 10 to $20 \%$ of children with leukemia. Musculoskeletal pain causing night-time awakening was reported by 5 of 6 patients with leukemia but not in any patients with sJRA. Non-articular bone pain was present in all patients with acute leukemia but in no patients with sJRA. Lymphadenopathy, hepatomegaly, and splenomegaly were present equally in the two groups. In leukemia, arthritis involved few joints and was more painful and temporary. On the other hand, in sJRA arthritis was persistent and additive. Among 10 patients with leukemia, seven had normal platelet counts and three had thrombocytosis; while among the 10 children with sJRA, seven had thrombocytosis. ESR was not diagnostically helpful, since it was elevated in all patients. At diagnosis two patients with leukemia still had thrombocytosis, but three developed thrombocytopenia. In sJRA, eight of 10 children had thrombocytosis. In sJRA, neutrophilia was uniformly present, while in leukemia lymphocytosis predominated. Moreover, four of 10 patients with leukemia had a leukocyte count $<4,500 / \mu$ l. Cabral et. al. [16] studied 29 children who were eventually diagnosed with malignancy (13 with leukemia) and were referred to two pediatric rheumatology centers. Patients had features typical of many rheumatic disorders. Features that were suggestive of malignancy included non-articular bone pain and abnormal initial complete blood count/ blood smear, elevated LDH and discordant erythrocyte sedimentation rate and platelet count, i.e., elevated ESR with normal or low platelets. Marwaha et. al. [17] studied 49 children with ALL initially misdiagnosed as JRA and 698 children with ALL that were properly diagnosed. The first group had significantly longer interval of symptoms prior to diagnosis ( $91 \pm 20$ days versus $54.6 \pm 4$ days), higher hemoglobin values $(8.09 \pm 0.8 \mathrm{~g} / \mathrm{dl}$ versus $7.03 \pm 0.2$ $\mathrm{g} / \mathrm{dl}$ ) and lower total leukocyte counts at presentation $\left(23.6 \pm 9.6 \times 10^{9} / \mu \mathrm{l}\right.$ versus $\left.48.7 \pm 6.26 \times 10^{9} / \mu \mathrm{l}\right)$. Additionally, the platelet count was borderline higher in children with ALL initially misdiagnosed as JRA compared to the remaining children with ALL (91 \pm $32.6 \times 10^{3} / \mu \mathrm{l}$ versus $\left.64.9 \pm 7.8 \times 10^{3} / \mu \mathrm{l}\right)$. Finally, Alter et. al. [18] reported that among 39 children with ALL, $70 \%$ had macrocytosis and/or anisocytosis at initial presentation. Our patient did not have either, since the MCV (71 fl) was below the $3^{\text {rd }}$ percentile for age, and moreover the RDW (14.2\%) was not indicative of anisocytosis.

ALL misdiagnosed as JRA may have serious consequences, if corticosteroids are administered for the presumed rheumatic disease, since single agent prednisone leads to a complete but transient remission approximately $60 \%$ of children with ALL [19]. Unfortunately, the pre-treated children recur with more resistant disease. This was shown in a Hungarian study of 699 children with ALL, 34 of whom had received prednisolone therapy prior to diagnosis because they were mistakenly considered as suffering from aplastic anemia or arthritic disorders. Although they entered complete remission at the same rate as the remaining children, the length of remission was significantly shorter than in the non-pretreated group [20].

\section{CONCLUSION}

Paediatricians and also orthopaedic surgeons and physicians of other medical specialties who examine 
children with musculoskeletal complaints should have a high index of suspicion for underlying malignancies, especially ALL, if there are abnormalities on the hemogram, even in the absence of circulating lymphoblasts. Pointers to leukemia include bone tenderness or pain, especially if nocturnal and nonarticular, anemia of any degree, low-normal leukocytes with relative lymphocytosis, red cell macrocytosis, and low-normal platelet counts, especially when associated with elevated ESR and increased serum LDH. Therefore, we recommend a pediatric hematologist to be involved early in the assessment of children with atypical musculoskeletal complaints and even slight abnormalities in their blood counts.

\section{$* * * * * * * * *$}

\section{Acknowledgement}

The authors would like to thank Dr. I. Kotsianidis for performing the flow cytometry.

\section{Author Contributions}

Elpis Mantadakis - Conception and design, Acquisition of data, Analysis and interpretation of data, Drafting the article, Final approval of the version to be published

Anastassios Valsamidis - Conception and design, Acquisition of data, Analysis and interpretation of data , Drafting of the article, Final approval of the version to be published

Athanassios Chatzimichael - Conception and design, Acquisition of data, Analysis and interpretation of data , Drafting of the article, Final approval of the submitted version

\section{Guarantor}

The corresponding author is the guarantor of submission.

\section{Conflict of Interest}

Authors declare no conflict of interest.

\section{Copyright}

(C) Elpis Mantadakis et. al. 2010; This article is distributed under the terms of Creative Commons attribution 3.0 License which permits unrestricted use, distribution and reproduction in any means provided the original authors and original publisher are properly credited. (Please see www.ijcasereportsandimages.com/copyrightpolicy.php for more information.)

\section{REFERENCES}

1. Pui CH, Relling MV, Downing JR. Acute lymphoblastic leukemia. $N$ Engl $\mathrm{J}$ Med 2004;350:1535-1548.

2. Smith OP, Hann IM. Clinical features and therapy of lymphoblastic leukemia. In: Arceci RJ, Hann IM, Smith OP, eds. Pediatric Hematology. $3^{\text {rd }}$ ed. London: Blackwell Publishing 2006: 450-481.

3. Miller ML. Evaluation of suspected rheumatic disease. In: Behrman RE, Kliegman RM, Jenson HB, eds. Nelson Textbook of Pediatrics. $17^{\text {th }}$ ed. Philadelphia: Saunders 2004: 793-795.

4. Miller ML, Scher I, Yogev R, Bernstein B. Fever of unknown origin. Pediatr Clin North Am 1995;42:999-1015.

5. Bradlow A, Barton C. Arthritic presentation of childhood leukaemia. Postgrad Med J 1991;67:562-564.

6. Murray MJ, Tang T, Ryder C, Mabin D, Nicholson JC. Childhood leukaemia masquerading as juvenile idiopathic arthritis. BMJ 2004;329:959-961.

7. Schaller J. Arthritis as a presenting manifestation of malignancy in children. J Pediatr 1972;81:793797.

8. Smith MA, Gloeckler Ries LA, Gurney JG, Ross JA. Leukemia: SEER pediatric monograph. In: Ries LAG, Smith MA, Gurney JG et al, eds. Cancer incidence and survival among children and adolescents: United States SEER Program 19751995, National Cancer Institute, SEER Program. Pub No. 99-4649. Bethesda: NIH, 1999.

9. Andersson Gäre B, Fasth A. Epidemiology of juvenile chronic arthritis in Southwestern Sweden: a 5-year prospective population study. Pediatrics 1992;90:950-988.

10. Wallendal M, Stork L, Hollister JR. The discriminating value of serum lactate dehydrogenase levels in children with malignant neoplasms presenting as joint pain. Arch Pediatr Adolesc Med 1996;150:70-73.

11. Chudwin DS, Ammann AJ, Cowan MJ, Wara DW. Significance of a positive antinuclear antibody test in a pediatric population. Am $\mathrm{J}$ Dis Child 1983;137:1103-1106.

12. Jones OY, Spencer CH, Bowyer SL, Dent PB, Gottlieb BS, Rabinovich CE. A multicenter casecontrol study on predictive factors distinguishing childhood leukemia from juvenile rheumatoid arthritis. Pediatrics 2006;117:e840-844.

13. Blatt J, Penchansky L, Horn M. Thrombocytosis as a presenting feature of acute lymphoblastic leukemia in childhood. Am J Hematol 1989;31:4649.

14. Jonsson OG, Sartain P, Ducore JM, Buchanan GR Bone pain as an initial symptom of childhood acute lymphoblastic leukemia: association with nearly normal hematologic indexes. $J$ Pediatr 1990;117:233-237.

15. Ostrov BE, Goldsmith DP, Athreya BH. Differentiation of systemic juvenile rheumatoid arthritis from acute leukemia near the onset of disease. J Pediatr 1993;122:595-598.

16. Cabral DA, Tucker LB. Malignancies in children who initially present with rheumatic complaints. J Pediatr 1999;134:53-57.

17. Marwaha RK, Kulkarni KP, Bansal D, Trehan A. Acute lymphoblastic leukemia masquerading as juvenile rheumatoid arthritis: diagnostic pitfall and association with survival. Ann Hematol 2010;89:249-254.

18. Alter BP, Weiner MA, Harris MB. Erythrocyte characteristics in childhood acute leukemia. Am J Pediatr Hematol Oncol 1989;11:8-15. 
19. Gaynon PS, Lustig RH. The use of glucocorticoids in acute lymphoblastic leukemia of childhood. Molecular, cellular, and clinical considerations. J Pediatr Hematol Oncol 1995;17:1-12.

20. Révész T, Kardos G, Kajtár P, Schuler D. The adverse effect of prolonged prednisolone pretreatment in children with acute lymphoblastic leukemia. Cancer 1985;55:1637-1640. 